\title{
Mit welcher Strategie ist das Risiko für einen Switch in eine Hypomanie am geringsten?
}

Fragestellung: Besteht bei depressiven Patienten mit Bipolar-IIStörung ein höheres Switch-Risiko in eine Hypomanie bei einer Monotherapie mit Sertralin im Vergleich zu einer Monotherapie mit Lithium oder einer Kombinationstherapie aus Lithium und Sertralin?

Hintergrund: Die Hypomanie wird von Patienten mit BipolarII-Störung oft als nicht behandlungsbedürftig angesehen und ist häufig unterdiagnostiziert. Es gibt Studien, die belegen, dass bei einer Bipolar-I-Störung durch eine reine antidepressive Therapie die Gefahr für einen Switch besteht. Aktuell gibt es nur wenige Daten zur Behandlung von Patienten mit Bipolar-II-Störung.

Patienten und Methodik: Es wurde eine randomisierte, kontrollierte Doppelblindstudie über 16 Wochen an vier Standorten in den USA durchgeführt. Die Rekrutierung erfolgte von Juli 2006 bis Juni 2013 über ambulante Einrichtungen. Die 142 Studienteilnehmer wurden als geschichtete Zufallsstichprobe auf die drei Therapien verteilt. Die minimale Zieldosis betrug $100 \mathrm{mg}$ Sertralin pro Tag und $900 \mathrm{mg}$ Lithium pro Tag. Der Zielbereich des Lithium-Spiegels lag zwischen 0,8-1,2 mmol/l. Die Studienteilnehmer soll-

Altshuler LL, Sugar CA, McElroy SL et al. Switch rates during acute treatment for bipolar II depression with lithium, sertraline, or the two combined: a randomized double-blind comparison AmJ Psychiatry 2017; 174: 266-76 ten zwischen 18 und 65 Jahren sein, die DSM-Kriterien für eine bipolare Störung erfüllen und aktuell eine depressive Episode aufweisen. Eine Hypomanie wurde durch mindestens 12 Punkte in der Young Mania Rating
Scale und einem Mania Severity Score von 2 oder 3 in der Clinical Global Impression Scale für bipolare Störungen definiert.

Ergebnisse: Es zeigte sich ein Switch-Risiko (in eine Hypomanie) von $17,9 \%$ in 16 Wochen, wobei kein signifikanter Unterschied zwischen den drei Therapien vorlag. $75 \%$ der Switches traten in den ersten fünf Wochen auf. Aufgefallen war, dass Patienten mit einem rapid cycling bei allen Therapiegruppen ein ähnliches Therapieansprechen zeigten, wohingegen die Patienten ohne ein rapid cycling ein höheres Therapieansprechen bei den beiden Monotherapien aufwiesen. Auch hinsichtlich der Häufigkeit therapiebedingter Nebenwirkungen unterschieden sich die drei Therapien nicht signifikant. Die Abbruchrate hingegen war signifikant höher bei der Kombinationstherapie. Es zeigte sich, dass die Teilnehmer mit einem Switch einen signifikant niedrigeren Lithium-Spiegel $(0,41 \mathrm{mmol} / \mathrm{l})$ verglichen $\mathrm{zu}$ den Teilnehmern ohne Switch (0,63 mmol/l) aufwiesen. Aufgefallen war zudem, dass Patienten mit einem Drogenmissbrauch/abhängigkeit in der Vorgeschichte ein erhöhtes Risiko für einen Switch und Patienten mit einem Alkoholmissbrauch/-abhängigkeit in der Vorgeschichte ein signifikant höheres Risiko für ein geringeres Therapieansprechen zeigten.

Schlussfolgerungen: Es besteht bei der Monotherapie mit Sertralin, der Monotherapie mit Lithium und der Kombinationstherapie aus Lithium und Sertralin ein gleich hohes SwitchRisiko. Das Therapieansprechen bei den rapid cyclern ist bei allen drei Therapieverfahren gleich, wohin gegen das Therapieansprechen bei Nicht-rapid-cyclern bei den beiden Monotherapien höher ist.

\section{- Kommentar von Eva Müller, Erlangen}

\section{Geringe Unterschiede zwischen den verglichenen Therapien}

Die randomisierte klinische Studie erfüllt mit einem JadadScore von 4 Punkten hohe methodische Ansprüche. Die hier vorgestellte Arbeit ist ein wichtiger Beitrag zum Vergleich verschiedener medikamentöser Therapien bei Bipolar-II-Störung. Die Untersuchung zeigte, dass es zwischen den drei Behandlungsmöglichkeiten nur geringe Unterschiede gibt. Die meisten Patienten mit einen Switch in eine Hypomanie erfuhren diesen in den ersten fünf Wochen, weshalb besonders zu Beginn der Therapie eine engmaschige Therapieüberwachung stattfinden sollte. Anders als erwartet zeigt sich bei der Kombinationstherapie kein früheres Therapieansprechen. Schwierig erscheint die Beurteilung der Therapie mit Lithium, da bei vielen Patienten die angestrebte Dosierung von $0,8-1,2$ $\mathrm{mmol} / \mathrm{I}$ nicht erreicht wurde. Da es derzeit nur sehr wenige Da- ten bezüglich der Rate an Patienten mit rapid cycling bei Bipolar-Il-Störung gibt, erscheint es fraglich, ob die Studie (hier $41,6 \%$ rapid cycler) diesbezüglich sehr repräsentativ ist.

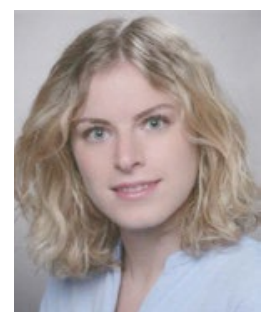

Eva Müller, Erlangen

Psychiatrische und Psychotherapeutische Klinik, Universitätsklinikum Erlangen E-Mail: eva.mueller@uk-erlangen.de 